\title{
Elementary processes with atoms and molecules in isolated and aggregated states ${ }^{\star}$
}

\author{
F. Aumayr ${ }^{1}$, K.H. Becker ${ }^{2}$, B. Marinković ${ }^{3}$, Štefan Matejčík ${ }^{4}$,a , and J. Tanis ${ }^{5}$ \\ 1 Institute of Applied Physics, Vienna University of Technology, Wiedner Hauptstr. 8-10/E134, 1040 Vienna, Austria \\ 2 Dept. of Applied Physics, New York University Polytechnic School of Engineering, 6 MetroTech Center, Brooklyn, \\ NY 11201, USA \\ 3 Institute of Physics, University of Belgrade, Pregrevica 118, 11080 Belgrade, Serbia \\ 4 Department of Experimental Physics, Comenius University, Mlynská dolina F2, 84248 Bratislava, Slovakia \\ 5 Department of Physics, Western Michigan University, 1903 W. Michigan Ave., 49008-5252 Kalamazoo, USA
}

Received 21 May 2015

Published online 7 July 2015 - (C) EDP Sciences, Società Italiana di Fisica, Springer-Verlag 2015

\begin{abstract}
This topical issue on "Elementary processes with atoms and molecules in isolated and aggregated states" was motivated by the 6th Conference on Elementary Processes in Atomic Systems - CEPAS, which was held from July 9-12, 2014 in Bratislava, Slovakia. The conference program overlaps with the topic of this issue. The scientific papers in the topical issue cover the fields of electron, positron, ion and photon interactions with atoms, molecules and surfaces.
\end{abstract}

\section{Introduction}

The term "elementary processes" on the atomic and molecular scale comprises a wide range of interactions of photons, electrons, ions, excited species and radicals with atoms and molecules in the gas phase. In addition to elementary processes with isolated particles (atoms and molecules) in many environments elementary processes with aggregated particles (clusters, nano-droplets, aerosols) and with surfaces of macroscopic objects and/or dust particles play an important role. Knowledge of the mechanisms and the kinetics of the elementary processes are of great importance for the understanding of macroscopic systems and for the development of predictive models. Likewise, understanding elementary processes at the atomic and molecular level is important in different fields of the science and technology, e.g., in plasmas and electric discharges [1], nanotechnology [2] processes in planetary atmospheres [3], radiation physics and chemistry [4].

Several conferences series cover the field of elementary processes at the atomic and molecular level (ICPEAC, ECAMP, EGAS...) and the Conference on Elementary Processes in Atomic Systems (CEPAS) also belongs to this family. The CEPAS conference series started 2000 in Uzhorod, Ukraine and the 6th CEPAS took place in Bratislava, Slovakia [5] where it was organized by the Department Experimental Physics, Comenius University

\footnotetext{
* Contribution to the Topical Issue "Elementary Processes with Atoms and Molecules in Isolated and Aggregated States", edited by Friedrich Aumayr, Bratislav Marinkovic, Stefan Matejcik, John Tanis and Kurt H. Becker.

${ }^{a}$ e-mail: matejcik@fmph.uniba.sk
}

with Professor Štefan Matejčík as the conference chair. The 93 scientific participants, 19 of whom were students, had many fruitful discussions and exchanges that contributed to the success of the conference and gave possibilities for new collaborations. Participants from 23 countries made the conference truly international in scope. The rather small size of the conference, and the informal nature of it, promoted the many discussions and led to much collegiality among the participants.

\section{Topics covered}

The topics of the conference covered six fields: (i) photo-induced processes and laser collisions; (ii) electron(positron)/atom collisions; (iii) collisions with biomolecules; (iv) heavy particles (ion/atom) collisions; (v) interactions with surfaces; and (vi) processes with nanosized complex systems.

The EPJD topical issue: "Elementary processes with atoms and molecules in isolated and aggregated states" contains contributions based on topics discussed at the conference.

A total of 29 papers were presented and 66 abstracts were contributed in poster sessions, providing a snap shot of the current research in the field of the elementary processes of electrons, positrons, photons and ions with atoms, molecules and surfaces. Of the 29 presented papers, 6 were invited lectures covering different areas of the conference: Dragana Maric (Institute of Physics, Belgrade) presented a talk on atomic and molecular processes in discharges in liquids, Paul Scheier (University Innsbruck) talked on the decoration of fullerene ions with simple molecules, Fernando Martin (University of Madrid) spoke 
about electron and nuclear dynamics in the photoionisation of molecules, Yasuyuki Nagashima (University of Science, Tokyo) discussed new developments in PS-studies, Don Madison (University of Missouri-Rolla) talked about accuracy of the theory of electron-impact ionization of molecules, and John Tanis (Western Michigan University, Kalamazoo) presented recent work on the transmission of electrons through micrometer-sized glass capillaries. In addition, there were 23 progress reports of ongoing current research, several of which included student speakers.

Contributed abstracts were presented in two afternoon sessions. These contributed papers divided between the main conference topics, formed an important part of the conference and provided ample opportunity for discussions. The posters were distributed among the two days of the conference so that approximately equal numbers of abstracts in the different areas were scheduled for each day.

The largest group of the papers in this topical issue is devoted to the topic of electron ionisation of atoms and molecules. The theoretical papers from this topic addressed the problem of kinetics of electron ionisation processes as manifested in the shape of the cross sections for the hydrogen atom and the "quasi oneelectron" alkali atoms [6], triple differential cross sections of $\mathrm{H}_{2} \mathrm{O}$ [7] and the mechanism of ionisation reactions [8]. The group of experimental papers included mainly mass spectrometric studies of electron ionisation processes, including the measurement of the mass spectra and ion efficiency curves for electron ionisation and dissociative ionisation with an emphasis on the identification of the reaction products for molecules such as sorbitol [9], $\mathrm{SF}_{6}$ [10], Tryptophan [11] and iron pentacarbonyl [12]. Mass spectra and thermochemical data such as reaction enthalpies and bond dissociation energies are reported as potential applications of the results reported in these papers which include the areas of plasmas, radiation chemistry and nanotechnology.

Another class of electron interactions addressed in the topical issue are inelastic electron scattering processes with atoms and molecules such as exploration of the singly and doubly excited states of neon at high incident electron energies [13] as well as two papers dealing with electron impact excitation studies producing emission spectra of $\mathrm{Se}_{n}$ and $\mathrm{Fe}(\mathrm{CO})_{5}[14,15]$. The interaction of positron interactions with atoms is represented by one paper dealing with the theoretical calculation of the cross section for $\mathrm{X}$ ray production from the L-shell excitation of $\mathrm{Ag}$, In and Sn atoms after positron impact [16]. The authors applied the binary-encounter approximation and the classical trajectory Monte Carlo methods and compared the results with the experimental and quantum-chemical data.

Photon processes were represented by two photoionisation studies. The first paper reports theoretical studies of the photoionisation of the $\mathrm{C}_{2}^{+}$ion in Rydberg states [17] using a multi-configuation Dirac-Fock approach with configuration interaction. The second study deals with ionisation of the $\mathrm{H}$ atom by intense two-cycle laser pulses [18]. The authors simulated the intra-pulse interference effects, and their dependence on the carrier-envelope phase of the laser pulse.
The last class of elementary processes included in this issue addresses the interaction of ions with atoms and molecules from a theoretical point of view and an experimental paper describes collisions of heavy ions with surfaces. The process of twoelectron exchange between anions and polar molecules was studied by Karbovanets et al. [19]. The transport properties of ions in water vapour area hot topic from the point of view of atmospheric pressure plasmas. The $\mathrm{O}$ transport in $\mathrm{H}_{2} \mathrm{O}$ vapour was the subject of a Monte Carlo simulation by Stojanovic et al. [20]. A new method was proposed for testing the effect of the projectile beam coherence on single ionization processes by calculating fully differential cross sections with quantum mechanical and impact parameter approximations. The theoretical results were compared for $\mathrm{C}^{6+}$ projectiles interacting with $\mathrm{He}$ atoms Ion surface interactions were studied by means of X-ray emission spectra following heavy inert ion (Ar $220 \mathrm{keV}$ ) impact on Fe/Si and $\mathrm{Fe} / \mathrm{Cu} / \mathrm{Si}$ thin films by Antoszewska-Moneta et al. [21]. The evaluation of the experimental data shows that the structure and composition of the thin films were modified by the ion irradiation and that the process can be monitored by particleinduced X-ray emissions (PIXE).

The chemistry of tungsten ions attracted attention in last years due to application of tungsten as wall material in International Thermonuclear Experimental Reactor (ITER). The recombination of the electrons with tungsten ions is an important process in high temperature plasma in case of contamination of plasma by tungsten. One of the recombination reaction $(\mathrm{W} 37+)$ was treated in the paper by $\mathrm{Wu}$ et al. [22] by ab initio methods yielding rate coefficient for this reaction.

\section{Epilogue}

All in all, the 6th CEPAS was very successful and greatly appreciated by the attendees. The invited lectures, the progress reports and the posters bridged the gap between the different fields of the conference, making it possible for non-experts in a given area to gain insight into a new area. It is noteworthy that among the speakers were several young scientists, specifically, postdocs and students, who brought new perspectives to their fields. The next CEPAS will take place in the Czech Republic in 2017 with the exact location and date still to be announced. Given the rapidity with which physics is advancing in all of the areas covered by CEPAS, we expect that future CEPAS conferences will be as stimulating as this most recent one was, as indicated by the contributions presented in this proceedings volume.

\section{References}

1. A. Friedman, Plasma Chemistry (Cambridge University Press, 2008)

2. I. Utke, S. Moshkalev, P. Russell, Nanofabrication Using Focused Ion and Electron Beams: Principles and Applications (Oxford University Press, 1. 5. 2012) 
3. J.T. Houghton, The Physics of Atmospheres (Cambridge University, Press, 1977)

4. E.B. Podgorsak, Radiation Physics for Medical Physicists (Biological and Medical Physics, Biomedical Engineering), 2nd edn. (Springer, 2010)

5. 6th Conference on Elementary Processes in Atomic Systems - CEPAS 2014, 9th-12th July 2014, Bratislava, Slovakia, http://neon.dpp.fmph.uniba.sk/cepas2014

6. H. Deutsch, K. Becker, Eur. Phys. J. D 69, 9 (2015)

7. I. Tóth, L. Nagy, R.I. Campeanu, Eur. Phys. J. D 68, 369 (2014)

8. B. Paripás, B. Palásthy, G. Pszota, Eur. Phys. J. D 69, 39 (2015)

9. I. Chernyshova, P. Markush, A. Zavilopulo, O. Shpenik, Eur. Phys. J. D 69, 80 (2015)

10. Sh.Sh. Demesh, E.Yu. Remeta, Eur. Phys. J. D 69, 168 (2015)

11. J. Tamuliene, L.G. Romanova, V.S. Vukstich, A.V. Papp, A.V. Snegursky, Eur. Phys. J. D 69, 21 (2015)

12. M. Lacko, P. Papp, K. Wnorowski, Š. Matejčík, Eur. Phys. J. D 69, $84(2015)$
13. J.J. Jureta, B. Marinković, A. Milosavljević, L. Avaldi, Eur. Phys. J. D 69, 74 (2015)

14. M. Erdevdy, P. Markush, O. Shpenik, V. Zvenihorodsky, Eur. Phys. J. D 69, 17 (2015)

15. A. Ribar, M. Danko, J. Országh, F. Ferreira da Silva, I. Utke, Š. Matejčík, Eur. Phys. J. D 69, 117 (2015)

16. T. Mukoyama, K. Tökési, Y. Nagashima, Eur. Phys. J. D 68, $342(2014)$

17. V. Stancalie, Eur. Phys. J. D 68, 349 (2014)

18. A. Tóth, S. Borbély, K. Tökési, L. Nagy, Eur. Phys. J. D 68, 349 (2014)

19. O.M. Karbovanets, M.I. Karbovanets, M.V. Khoma, V.Y. Lazur, Eur. Phys. J. D 68, 11 (2014)

20. V. Stojanović, Z. Raspopović, D. Marić, Z.Lj. Petrović, Eur. Phys. J. D 69, 63 (2015)

21. M. Antoszewska-Moneta, R. Brzozowski, M. Moneta, Eur. Phys. J. D 69, 77 (2015)

22. Z. Wu, Y. Zhang, Y. Fu, A. Surzhykov, S. Fritzsche, C. Dong, Eur. Phys. J. D 69140 (2015) 